\title{
INDEX CHARACTER ASSOCIATED WITH THE PROJECTIVE DIRAC OPERATOR
}

\author{
MAKOTO YAMASHITA \\ (Communicated by Varghese Mathai)
}

\begin{abstract}
We calculate the equivariant index formula for an infinite dimensional Clifford module canonically associated with any closed oriented Riemannian manifold. It encompasses the fractional index formula of the projective Dirac operators by Mathai-Melrose-Singer. Our method can be regarded as the calculation of a topological index for such operators.
\end{abstract}

\section{INTRODUCTION}

In their pioneering paper MMS06, Mathai, Melrose, and Singer defined the notion of projective vector bundles and pseudodifferential operators on such bundles extending the notion of classical pseudodifferential operators. This new framework ignited various developments of the study of modules over the bundles of matrix algebras and index theory for such modules [MMS05, MMS09, AM, BG, Gof10].

In the most fundamental setting of [MMS06] they gave the projective Dirac operator $\not_{M}^{\mathrm{pr}}$ canonically associated with the arbitrary closed oriented Riemannian manifold $M$. Although this object cannot be represented as a Fredholm operator on a Hilbert space in general, they defined its index $\operatorname{ind}_{a} \not \partial_{M}^{\mathrm{pr}}$ in terms of the integral kernel $I_{\not{ }_{M}}^{\mathrm{pr}}$ and showed the fractional index formula [MMS06, Theorem 2 and Section 9]:

$$
\operatorname{ind}_{a} \not \partial_{M}^{\mathrm{pr}}=\int_{M} \hat{A}\left(R_{M}\right) .
$$

Here, the integrand of the right hand side is the $\hat{A}$-form associated with the LeviCivita connection of $M$. The equality was proved via the local asymptotic expansion of the heat kernel associated with $I_{\not{ }_{M}}^{\mathrm{pr}}$. It can easily be seen from the above formula that the index of the projective Dirac operator can be a non-integral rational number when $M$ is not a spin manifold.

The projective operator $\partial_{M}^{\mathrm{pr}}$ is associated with a certain Clifford module of $M$, but one has to note the difference between (11) and the usual index formula of the twisted Dirac operator $\not_{E}$ associated with a Clifford module $E$ over $M$ given by

$$
\text { ind } \not_{E}=\int_{M} \hat{A}\left(R_{M}\right) \operatorname{ch}(E / \mathbb{S}) \text {. }
$$

Received by the editors February 3, 2011 and, in revised form, November 10, 2011.

2010 Mathematics Subject Classification. Primary 58J22; Secondary 35K05.

Key words and phrases. Twisted index theory, Clifford module.

This work has been supported by the Marie Curie Research Training Network MRTN-CT2006-031962 in Noncommutative Geometry, EU-NCG. 
Here, the relative Chern character $\operatorname{ch}(E / \mathbb{S})$ of $E$ comes into play and makes the integral on the right hand side an integer.

Suppose that the dimension of $M$ is an even integer $n$, and let us identify the automorphism group of the Clifford algebra of $\mathbb{R}^{n}$ with $\mathrm{PU}(N)$ for a suitable integer $N$. By induction from the frame bundle we obtain a natural $\mathrm{PU}(N)$-principal bundle $P$ over $M$. Moreover, let $\pi^{(\text {nat })}: \mathrm{SU}(N) \curvearrowright V^{(\text {nat })}$ be the natural representation of $\mathrm{SU}(N)$. The Levi-Civita connection on $F_{\mathrm{SO}(n)}$ induces a 1-form $\nabla$ of first order differential operators acting on the space $C^{\infty}\left(P, V^{(n a t)}\right)$. This means, for each vector field $X$ over $M$, one obtains a first order differential operator $\nabla_{X}$ on $P$.

Since $P$ can be regarded as the bundle of trivializations of the Clifford bundle $\mathrm{Cl}_{\mathbb{C}}(M)$, the sections of $\mathrm{Cl}_{\mathbb{C}}(M)$ can be identified with the $\mathrm{PU}(N)$-invariant functions of $P$ into $M_{N}(\mathbb{C})$. Then it can be seen that the space of functions from $P$ into $V^{(\text {nat) }}$ admits a structure of the module over $\mathrm{Cl}_{\mathbb{C}}(M)$. Thus one may define the associated Dirac operator

$$
\not_{M}=\sum_{k} c\left(e_{k}\right) \nabla_{e_{k}}
$$

acting on $C^{\infty}\left(P, V^{(\text {nat })}\right)$, where $\left(e_{i}\right)_{i=1}^{n}$ is any local frame of $M$.

The operator $\not_{M}$ is a transversely elliptic operator Ati74] on the $\mathrm{SU}(N)$-manifold $P$. Hence one obtains a distribution $\operatorname{ind}_{\mathrm{SU}(N)}\left(\gamma, \not_{M}\right)$ on $\mathrm{SU}(N)$ which is invariant under conjugation and satisfies

$$
\left\langle\operatorname{ind}_{\mathrm{SU}(N)}\left(\gamma, \not \partial_{M}\right), \chi^{\pi}\right\rangle=\operatorname{ind}\left(\not \not_{M} p^{\pi}\right) .
$$

Here, $\chi^{\pi}$ is the character of any irreducible representation $\pi$ of $\mathrm{SU}(N)$ and $p^{\pi}$ is the projector onto the $\pi$-isotypic component of the action $\pi^{P} \otimes \pi^{(\text {nat })}$ on $C^{\infty}\left(P, V^{(\text {nat })}\right)$, where $\pi^{P}$ is the action induced by the translation on $P$.

In MMS08, it was shown that the $\mathrm{SU}(N)$-equivariant operator $\not \partial_{M}$ descends to the projective Dirac operator $\not \partial_{M}^{\mathrm{pr}}$ on the projective spin bundle over $\mathrm{Cl}_{\mathbb{C}}(M)$. It was then observed that

$$
\left\langle\operatorname{ind}_{\mathrm{SU}(N)}\left(\gamma, \not_{M}\right), \phi\right\rangle=\operatorname{ind}_{a} \not_{M}^{\mathrm{pr}},
$$

where $\phi$ is any smooth test function on $\mathrm{SU}(N)$ which is constantly equal to 1 around $e$ and has small enough support. As mentioned at the end of the introduction of [MMS08], the fractional index formula gives the coefficient of the Dirac function of the distribution $\operatorname{ind}_{\mathrm{SU}(N)}\left(\gamma, \not_{M}\right)$.

The aim of this paper is to relate the fractional index formula (1) to the classical index formula (2) of Clifford modules and give a refined formula (Corollary 6) which also describes the contribution of higher order derivatives of the Dirac function in the distribution $\operatorname{ind}_{\mathrm{SU}(N)}\left(\gamma, \not_{M}\right)$.

Our starting point is that the Hilbert space $L^{2}\left(P, V^{(\text {nat })}\right)$ can be regarded as the space $\Gamma(M, \mathcal{E})$ of the sections of an infinite dimensional Clifford module $\mathcal{E}$ over $M=P / \mathrm{PU}(N)$. It is defined as the induced vector bundle

$$
\mathcal{E}=P \times_{\mathrm{PU}(N)} L^{2}(\mathrm{PU}(N)) \otimes V^{(\text {nat })},
$$

where we consider the left translation action $\lambda$ on $L^{2}(\mathrm{PU}(N))$ and the trivial action on $V^{\text {(nat) }}$. The precise correspondence between $L^{2}\left(P, V^{(\text {nat })}\right)$ and $\mathcal{E}$ is given by the 
equality

$$
L^{2}\left(P, V^{(\text {nat })}\right) \simeq\left(L^{2}(P) \otimes L^{2}(\mathrm{PU}(N)) \otimes V^{(\text {nat })}\right)^{\pi^{P} \otimes \lambda \otimes \operatorname{triv}_{V}(\text { nat })}(\mathrm{PU}(N)),
$$

where the right hand side admits a natural interpretation as $\Gamma(M, \mathcal{E})$.

The action $\rho \otimes \pi^{(\text {nat })}$ of $\mathrm{SU}(N)$ on $L^{2}(\mathrm{PU}(N)) \otimes V^{(\text {nat })}$ commutes with the one $\lambda \otimes$ triv of $\mathrm{PU}(N)$. Hence it induces an action of $\mathrm{SU}(N)$ on (4). This action corresponds to the action $\pi^{P} \otimes \pi^{(\text {nat })}$ on $L^{2}\left(P, V^{(\text {nat })}\right)$.

If one tries to consider an analogue of the equivariant index theorem in the case of $E=\mathcal{E}$, the relative curvature form $F^{\mathcal{E} / \mathbb{S}}$ of $\mathcal{E}$ shall be locally given by the action of the curvature of a vector bundle $\mathcal{E}_{0}$ satisfying $\mathcal{E} \simeq \mathcal{E}_{0} \otimes \mathbb{S}$. Note that the vector bundle $\mathcal{E}_{0}$ needs to have infinite rank, hence the fiberwise trace $\operatorname{Tr}_{\mathcal{E}_{0}} e^{-F^{\mathcal{E} / \mathbb{S}}}$ will become infinite. To remedy this, we shall construct an action of $\mathrm{SU}(N)$ on $\mathcal{E}_{0}$ such that its tensor product with the trivial action on $\mathbb{S}$ is the action on $\mathcal{E}$. Then an equivariant choice of curvature on $\mathcal{E}_{0}$ will allow us to make sense of the trace $\operatorname{Tr}_{\mathcal{E}_{0}}\left(e^{-F^{\mathcal{E} / \mathbb{S}}} \pi(\phi)\right)$ of the composition of the curvature $e^{-F}$ with the convolution by any auxiliary test function $\phi$ on $\mathrm{SU}(N)$.

Thus the expression

$$
\operatorname{ch}_{\mathrm{SU}(N)}(\gamma, \mathcal{E} / \mathbb{S})_{x}=\operatorname{Tr}_{\mathcal{E}_{0}}\left(\gamma e^{-F^{\mathcal{E} / \mathbb{s}}}\right)
$$

for the variable $x$ on $M$ and $\gamma$ on $\mathrm{SU}(N)$ defines a differential form on $M$ with coefficients in the distributions on $\mathrm{SU}(N)$. Then the analogue of the index formula (2) for the Clifford module $\mathcal{E}$ should be

$$
\operatorname{ind}_{\mathrm{SU}(N)}\left(\gamma, \not \partial_{M}\right)=\int_{M} \hat{A}\left(R_{M}\right) \operatorname{ch}_{\mathrm{SU}(N)}(\gamma, \mathcal{E} / \mathbb{S}) .
$$

In view of the correspondence (3), this gives a complete description of the 'topological index' of the projective Dirac operator of $M$.

\section{Preliminaries}

Most of the constructions in this section appear in the literature in some way or another. We just recall their definitions in order to fix the notation and conventions. For the sake of simplicity we assume that $n=\operatorname{dim}(M)$ is even and put $N=2^{n / 2}$. Let $P$ be the $\mathrm{PU}(N)$ principal bundle over $M$ induced from the frame bundle $F_{\mathrm{SO}(n)}$ by the natural group embedding

$$
\mathrm{SO}(n) \rightarrow \operatorname{Aut}\left(\mathrm{Cl}_{\mathbb{C}}\left(\mathbb{R}^{n}\right)\right) \simeq \mathrm{PU}(N) .
$$

Let $\sigma$ be a section of $F_{\mathrm{SO}(n)}$ defined on an open set $U$. It gives a trivialization of $\left.F_{\mathrm{SO}(n)}\right|_{U}$ and the associated trivialization $\left.P\right|_{U} \simeq U \times \mathrm{PU}(N)$. Let $\tilde{\sigma}=(\sigma, e)$ be the section of $P$ defined by $\sigma$ and the constant mapping $M \rightarrow \mathrm{PU}(N), x \mapsto e$. Under the identification of (4) any section $f$ of $\mathcal{E}$ over $U$ can be expressed using a function $\xi: U \ni x \mapsto \xi_{x} \in L^{2}\left(\mathrm{PU}(N), V^{(\text {nat })}\right)$ by

$$
f_{x} \leftrightarrow\left(\sigma_{x}, \xi_{x}\right)
$$

The Clifford module structure on $\mathcal{E}$ can be described as

$$
c(X) \cdot\left(\sigma_{x}, f_{x}\right)=\left(\sigma_{x}, \operatorname{Ad}_{g}\left(\sigma_{x}^{*}(c(X))\right) f_{x}(g)\right),
$$

where $\sigma_{x}^{*}$ is the isomorphism $\mathrm{Cl}_{\mathbb{C}}\left(\mathrm{T}_{x} M\right) \rightarrow M_{N}(\mathbb{C})$ given by $\sigma_{x} \in P_{x}$. 
Let $A^{\mathrm{LC}}$ be the Levi-Civita connection 1 -form $\mathrm{TF} \rightarrow \mathfrak{s o}(n)$ on $F_{\mathrm{SO}(n)}$. One obtains the induced connection form

$$
A: \mathrm{T} P \rightarrow \mathfrak{s u}(N)
$$

via (77). Hence it is given by the collection of linear maps $T_{p} P \rightarrow \mathfrak{s u}(N)$ which are retractions of the embedding $\mathfrak{s u}(N) \rightarrow T_{p} P$ coming from the action map $g \mapsto p . g$ from $\mathrm{SU}(N)$ to $P$ and which satisfy the equivariance condition $A_{X}=\operatorname{Ad}_{g} A_{g . X}$ for any $X \in T_{p} P$ and $g \in G$.

Given a vector field $X$ on $M$, define the operator $A_{X}^{(\mathcal{E}, \sigma)}$ on the sections of $\mathcal{E}$ over $U$ by

$$
A_{X}^{(\sigma)} \cdot\left(\tilde{\sigma}_{x}, \xi_{x}\right)=\left(\tilde{\sigma}_{x},\left(\lambda\left(A_{d \tilde{\sigma}\left(X_{x}\right)}\right) \otimes 1_{V^{(\mathrm{nat})}}\right)\left(\xi_{x}\right)\right) .
$$

Lemma 1. Let $\psi$ be a function from $U$ to $\mathrm{SU}(N)$. Then one has

$$
A_{X}^{(\sigma . \psi)}\left(\sigma . \psi_{x}, \psi_{x}^{-1} \xi_{x}\right)=\left(\sigma . \psi_{x}, \lambda\left(A_{d(\sigma)(X)}\right)^{\psi_{x}} \psi_{x} \xi_{x}-d \psi(X) . \psi_{x}^{-1} \cdot \xi_{x}\right)
$$

for any vector field $X$ on $U$.

By Lemma 1, the covariant derivative

$$
\left.\nabla_{X}\left(\sigma_{x}, \xi_{x}\right)=\left(\sigma_{x}, X\left(\xi_{x}\right)\right)+A_{X}^{(\sigma)} \cdot\left(\sigma_{x}, \xi_{x}\right)\right)
$$

associated with $A$ does not depend on the choice of $\sigma$. Moreover, formula (9) implies $\left[\nabla_{X}, c(Y)\right]=c\left(\nabla_{X}^{\mathrm{LC}} Y\right)$, which shows that $A$ is a Clifford connection. Finally, we put

$$
\not \partial_{M}=\sum_{i=1}^{n} c\left(e_{i}\right) \nabla_{e_{i}}
$$

for any local frame $\left(e_{i}\right)_{i=1}^{n}$.

For each irreducible representation $\pi$ of $\mathrm{SU}(N)$, the restriction of $\not \partial$ to the $\pi$ isotypic component of $L^{2}\left(P, V^{(\text {nat })}\right)$ becomes a $K$-cycle $\not_{\pi}$ over $C(P) \rtimes \operatorname{SU}(N)$. Hence we obtain a family of $K$-cycles $\left(\not_{\pi}\right)_{\pi \in \widehat{\mathrm{SU}(N)}}$ parametrized by the irreducible representations of $\mathrm{SU}(N)$. Since the $\pi$-isotypic component of $L^{2}\left(P, V^{(\text {nat })}\right)$ is trivial unless the central part of $\pi$ agrees with that of the natural representation, our in-

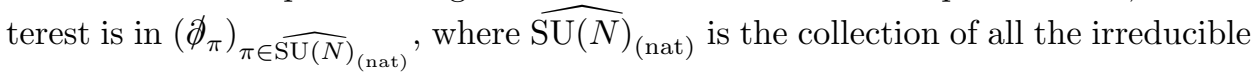
representation classes whose central character agrees with that $V^{(\text {nat })}$.

For each $\pi \in \widehat{\mathrm{SU}(N)}{ }_{(\text {nat })}$, the number ind $\not_{\pi}$ is finite and agrees with the multiplicity of $\pi$ in the representation ind $\partial_{M}$ of $\mathrm{SU}(N)$. Let $d_{\pi}$ be the dimension of the representation space of $\pi$ and $\chi^{\pi}$ be its normalized character

$$
\chi^{\pi}(\gamma)=\frac{1}{d_{\pi}} \operatorname{Tr}(\pi(\gamma))
$$

Then the sum

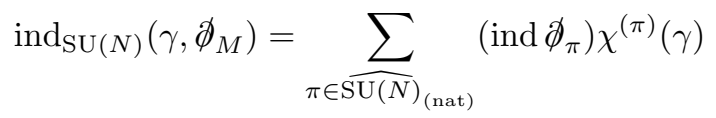

defines a distribution over $\mathrm{SU}(N)$ which is invariant under the conjugation. 
2.1. Pairing of $\mathcal{U}(\mathfrak{g})$ and $C^{\infty}(G)$. When $a$ is an element of the universal enveloping algebra $\mathcal{U}(\mathfrak{s u}(N))$ of $\mathrm{SU}(N)$, it can be regarded as a distribution on $\mathrm{SU}(N)$ with support $\{e\}$ by the formula

$$
\langle a, \phi\rangle=(\lambda(a) \phi)(e) \quad\left(\phi \in C^{\infty}(\mathrm{SU}(N))\right),
$$

where $\lambda(a)$ is the right invariant differential operator represented by $a$. The above pairing can also be expressed using the operator trace on $L^{2}(\mathrm{SU}(N))$ as

$$
\langle a, \phi\rangle=\operatorname{Tr}_{L^{2}(\operatorname{SU}(N))}(\lambda(a) \lambda(\phi))=\operatorname{Tr}_{L^{2}(\operatorname{SU}(N))}(\rho(a) \rho(\phi)) .
$$

Let $T$ be a conditional expectation of $\mathcal{U}(\mathfrak{s u}(N))$ onto its center.

Lemma 2. Let a be an element of $\mathcal{U}(\mathfrak{s u}(N))$ and $\phi$ an element in $C^{\infty}(\mathrm{SU}(N))$. Then one has

$$
\sum_{\pi \in \mathrm{SU}(N)} \operatorname{Tr}(\pi(a)) \operatorname{Tr}(\bar{\pi}(\phi))=\frac{1}{N} \sum_{g \in Z(\mathrm{SU}(N))} \chi^{(\mathrm{nat})}(g)\left\langle T(a), \lambda_{g} \phi\right\rangle .
$$

Proof. For each irreducible representation $\pi$ of $\mathrm{SU}(N)$, the operator $\pi(T(a))$ is a scalar satisfying $\operatorname{Tr}(\pi(a))=\operatorname{Tr}(\pi(T(a)))$. Hence one has

$$
\operatorname{Tr}_{L^{2}(\mathrm{SU}(N))}(\lambda(a) \rho(\phi))=\sum_{\pi \in \mathrm{SU}(N)} \operatorname{Tr}(\pi(a)) \operatorname{Tr}(\bar{\pi}(\phi))=\operatorname{Tr}_{L^{2}(\mathrm{SU}(N))}(\rho(T(a)) \rho(\phi)) .
$$

Combining this with

$$
\operatorname{Tr}_{L^{2}(\mathrm{SU}(N))_{\chi^{(\mathrm{nat})}}}(\lambda(a) \rho(\phi))=\frac{1}{N} \sum_{g \in Z(\mathrm{SU}(N))} \chi^{(\mathrm{nat})}(g) \operatorname{Tr}_{L^{2}(\mathrm{SU}(N))}\left(\lambda(a) \rho(\phi) \rho_{g}\right),
$$

one obtains

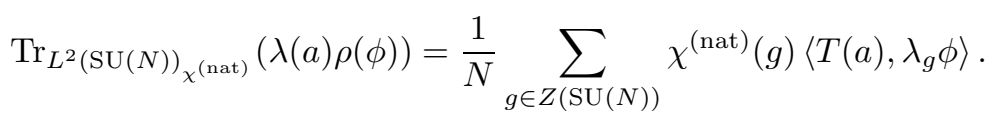

This proves the assertion.

\section{3. $K K$-element associated with projective Dirac operator}

For each character $\chi$ on the center of $\mathrm{SU}(N)$, let $J_{\chi}$ denote the closure of

$$
\{f \in C(\mathrm{SU}(N), C(P)) \mid \forall z \in Z(\mathrm{SU}(N)): f(g z)=\chi(z) f(g)\}
$$

in $C(P) \rtimes \mathrm{SU}(N)$. Then the algebra $C(P) \rtimes \mathrm{SU}(N)$ admits a direct sum decomposition

$$
C(P) \rtimes \mathrm{SU}(N) \simeq \bigoplus_{\chi \in Z \overline{(\mathrm{SU}(N)}} J_{\chi}
$$

by bilateral ideals. The representation of $C(P) \rtimes \mathrm{SU}(N)$ on $L^{2}\left(P, V^{(\text {nat })}\right)$ factors through the projection onto $J_{\text {(nat) }}$, the factor corresponding to the central character of the natural representation.

The bimodule $L^{2}\left(P, V^{(\text {nat })}\right)$ is a completion of the following $C^{*}$-module $\mathcal{F}$ over $\mathrm{Cl}_{\mathbb{C}}(M)$. The subspace $C\left(P, V^{(\text {nat })}\right)$ of $L^{2}\left(P, V^{(\text {nat })}\right)$ admits a $\mathrm{Cl}_{\mathbb{C}}(M)$-valued inner product characterized by

$$
p^{*}\left((\xi, \eta)_{x}\right)=\int_{\mathrm{PU}(N)} \operatorname{Ad}_{g}\left(\eta_{p} \otimes \xi_{p}^{*}\right) d g
$$


where we identify $p \in P_{x}$ with an algebra isomorphism $p^{*}: \mathrm{Cl}_{\mathbb{C}}\left(T_{x} M\right) \rightarrow M_{N}(\mathbb{C})$. The completion $\mathcal{F}$ of $C\left(P, V^{(\text {nat })}\right)$ with respect to the above inner product admits an action of $C(P) \rtimes \mathrm{SU}(N)$ as $\mathrm{Cl}_{\mathbb{C}}(M)$-compact operators.

Proposition 3. The bimodule $\mathcal{F}$ gives a strong Morita equivalence between $J_{(\text {nat })}$ and $\mathrm{Cl}_{\mathbb{C}}(M)$.

Each irreducible representation $\pi$ of $\mathrm{SU}(N)$ gives a class $[\pi]$ in $K_{0}\left(C^{*} \mathrm{SU}(N)\right)$. Under the inclusion $\iota: C^{*} \mathrm{SU}(N) \rightarrow C(P) \rtimes \mathrm{SU}(N)$, one obtains an element $\iota_{*}[\pi]$ in $K_{0}(C(P) \rtimes \mathrm{SU}(N))$. By Proposition 3 , one obtains an element $\iota_{*}[\pi]$ of $K_{0}\left(\mathrm{Cl}_{\mathbb{C}}(M)\right)$ given by the Clifford module $V_{\pi}=p_{\pi} L^{2}\left(P, V^{(\text {nat })}\right)$.

The operator $\not \partial_{M}$ and the representation of $C(P) \rtimes \mathrm{SU}(N)$ on $L^{2}\left(P, V^{(\text {nat })}\right)$ define a spectral triple over $C(P) \rtimes \mathrm{SU}(N)$. Consequently, we obtain the associated element $\alpha$ of $K K(C(M) \rtimes \mathrm{SU}(N), \mathbb{C})$ represented by the phase of $\not \partial_{M}$, as in [BC00], and the associated map

$$
\operatorname{ind}_{\not \not_{M}}: K_{0}(C(P) \rtimes \mathrm{SU}(N)) \simeq K_{0}\left(\mathrm{Cl}_{\mathbb{C}}(M)\right) \rightarrow \mathbb{Z} .
$$

Example 4. As an example of the above construction, consider the case of $\pi=$ $\pi^{\text {(nat) }}$. Then the corresponding index ind $\left(\not \partial_{\pi^{(n a t)}}\right)$ is equal to the signature number of $M$. Indeed, if one takes the tensor product action $\rho \otimes \pi \otimes \bar{\pi}$ on

$$
C\left(P ; V^{(\text {nat })}\right) \otimes\left(V^{(\text {nat })}\right)^{*},
$$

its fixed point subspace is identified with the space of the sections of $\mathrm{Cl}_{\mathbb{C}}(M)$, and the grading on the former corresponds to the left Clifford action of the volume element. Meanwhile the fixed point subspace is canonically identified with the

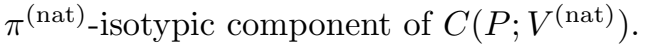

\section{ConneCtions on Infinite Dimensional EQUivariant BUNDleS}

Now we give a more precise description of (5). Let $A$ be the curvature 1-form of (10). The associated connection 2-form in $A^{2}(P, \mathfrak{s u}(N))_{\text {basic }}$ is given by

$$
\Omega_{X, Y}=A\left(\left[X-A_{X}, Y-A_{Y}\right]\right)
$$

for vector fields $X$ and $Y$ on $P$.

Let $\sigma$ be a section of $P \rightarrow M$ defined on an open set $U$ of $M$. When $X$ and $Y$ are vector fields on $M$, consider the operator

$$
\Omega_{X, Y}^{(\sigma)} \cdot\left(\sigma_{x}, \xi_{x}\right)=\left(\sigma_{x}, \lambda\left(\Omega_{d \sigma\left(X_{x}\right), d \sigma\left(Y_{x}\right)}\right) \xi_{x}\right)
$$

on the sections of $\mathcal{E}$ over $U$ as in (11). This is independent of the choice of $\sigma$, and the operators $\Omega_{X, Y}^{(\sigma)}$ on different open sets naturally patch together and give a 2-form of bundle morphisms $F_{X, Y}$ on $\mathcal{E}$.

Then $F_{X, Y}$ commutes with the action of $\rho \otimes \pi^{(\text {nat })}(\mathrm{SU}(N))$ and that of the Clifford algebra. Similarly we obtain $2 k$-forms of endomorphisms of $\mathcal{E}$ by the alternating product construction

$$
F_{X_{1}, \ldots, X_{2 k}}^{k}=\sum_{\sigma \in S_{2 k}}(-1)^{|\sigma|} F_{X_{\sigma 1}, X_{\sigma 2}} \cdots F_{X_{\sigma(2 k-1)}, X_{\sigma 2 k}} .
$$


4.1. Fell's absorption. Let $\phi$ be a smooth function on $\mathrm{SU}(N)$. Then the right convolution by $\phi$ on $P$ defines a trace class operator $\rho_{P} \otimes \pi^{(\text {nat })}(\phi)$ on $L^{2}\left(P, V^{(\text {nat })}\right)$. Thus one is interested in the computation of

$$
\operatorname{Tr}_{L^{2}\left(\mathrm{PU}(N) ; V^{(\text {nat })}\right)}\left(e^{-F^{\mathcal{E} / \mathrm{s}}} \rho \otimes \pi^{(\text {nat })}(\phi)\right) .
$$

Let us consider the following operator $S$ on $L^{2}\left(\mathrm{SU}(N) ; V^{(\text {nat })}\right)$. When $\phi$ is a function of SU(N) into $V^{(\text {nat })}$, we define $S \phi$ to be the function $(S \phi)(\gamma)=\pi^{(\text {nat })}(\gamma) \phi(\gamma)$. Then $S$ is an isomorphism of the $\mathrm{SU}(N)$-bimodule ${ }_{\lambda} L^{2}\left(\mathrm{SU}(N) ; V^{(\text {nat })}\right)_{\rho \otimes \pi^{(\text {nat })}}$ into $\lambda \otimes \pi^{(\text {nat })} L^{2}\left(\mathrm{SU}(N) ; V^{(\text {nat })}\right)_{\rho}$.

Then the submodule

$$
{ }_{\lambda} L^{2}\left(\mathrm{PU}(N) ; V^{(\text {nat })}\right)_{\rho \otimes \pi} \text { (nat) } \subset{ }_{\lambda} L^{2}\left(\mathrm{SU}(N) ; V^{(\text {nat })}\right)_{\rho \otimes \pi^{(\text {nat })}}
$$

is mapped to the subspace $L^{2}\left(\mathrm{SU}(N) ; V^{(\text {nat })}\right)^{\rho\left(\chi^{(\text {nat })}\right)}$ spanned by

$$
\left\{\phi \in L^{2}\left(\mathrm{SU}(N) ; V^{(\text {nat })}\right) \mid \forall \gamma \in Z(\mathrm{SU}(N)): \rho_{\gamma}(\phi)=\chi^{(\text {nat })}(\gamma) \phi\right\}
$$

of $\lambda \otimes \pi^{\text {(nat) }} L^{2}\left(\mathrm{SU}(N) ; V^{(\text {nat })}\right)_{\rho}$. This bimodule admits a direct sum decomposition

$$
L^{2}\left(\mathrm{SU}(N) ; V^{(\mathrm{nat})}\right)^{\rho\left(\chi^{(\mathrm{nat})}\right)}=\bigoplus_{\pi \in \mathrm{SU}(N)}{ }_{(\mathrm{nat})} \pi \otimes \pi^{(\mathrm{nat})}\left(V_{\pi} \otimes V^{(\mathrm{nat})}\right) \otimes\left(V_{\pi}\right)_{\bar{\pi}}
$$

Under the transformation $S$, the operator $\lambda\left(\Omega_{X, Y}^{(\sigma)}\right)$ is identified with the operator

$$
\lambda \otimes \pi^{(\mathrm{nat})}\left(\Omega_{X, Y}^{(\sigma)}\right)=\lambda\left(\Omega_{X, Y}^{(\sigma)}\right) \otimes 1_{V^{(\mathrm{nat})}}+1 \otimes \pi^{(\mathrm{nat})}\left(\Omega_{X, Y}^{(\sigma)}\right) .
$$

This shows that the Clifford module $\mathcal{E}$ can be decomposed locally as the tensor product $\mathcal{E}_{0} \otimes \mathbb{S}$, where $\mathcal{E}_{0}$ is a bundle of fiber $C(\mathrm{SU}(N))_{\chi^{(\text {nat })}}$ defined by

$$
\mathcal{E}_{0} \simeq P \times_{\mathrm{SU}(N)} C(\mathrm{SU}(N))_{\chi^{(\mathrm{nat})}} .
$$

4.2. Computation of index character. On one hand, the first part in the right hand side of (15) can be thought of as the action of the relative curvature form $F_{X, Y}^{\mathcal{E} / \mathbb{S}}=F_{X, Y}^{\mathcal{E}_{0}}$. On the other hand, the second part can be thought of as the action of the spin curvature $\sum_{i, j}\left\langle R_{X, Y}^{\mathrm{LC}} e_{i}, e_{j}\right\rangle c_{i} c_{j}$. Hence the quantity (13) can be translated to

$$
\operatorname{Tr}_{L^{2}\left(\mathrm{PU}(N) ; V^{(\text {nat })}\right)}\left(\lambda\left(e^{-\Omega}\right) \rho(\phi)\right)=N \operatorname{Tr}_{L^{2}(\mathrm{SU}(N))_{\chi}{ }^{\text {(nat) }}}\left(\lambda\left(e^{-\Omega}\right) \rho(\phi)\right) .
$$

Let $k$ be an integer. Combining (12) with (16), the $2 k$-form $\operatorname{Tr}_{\mathcal{E}}\left(\rho(\phi) F_{X_{1}, \ldots, X_{2 k}}^{k}\right)$ can be written as

$$
\sum_{\sigma \in S_{2 k}, g \in Z(\mathrm{SU}(N))}(-1)^{|\sigma|}\left\langle T\left(\Omega_{X_{\sigma 1}, X_{\sigma 2}}^{(\sigma)} \cdots \Omega_{X_{\sigma(2 k-1)}, X_{\sigma 2 k}}^{(\sigma)}\right), \lambda_{g} \phi\right\rangle .
$$

Consequently we obtain

$$
\left\langle\operatorname{Tr}_{\mathcal{E}}\left(\gamma e^{-F / \mathbb{S}}\right), \phi\right\rangle=\sum_{j=0}^{m / 2} \frac{1}{j !} \sum_{g \in Z(\mathrm{SU}(N))}\left\langle T\left(\left(\Omega^{(\sigma)}\right)^{j}\right), \lambda_{g} \phi\right\rangle,
$$

which gives the meaning as a distribution on $\mathrm{SU}(N)$ to the expression (5). 
Theorem 1. Let $\pi$ be an irreducible representation with central character $\chi^{(\mathrm{nat})}$. The distribution $\operatorname{ind}_{\mathrm{SU}(N)}\left(\gamma, \not \partial_{M}\right)$ satisfies

$$
\left\langle\operatorname{ind}_{\mathrm{SU}(N)}\left(\gamma, \not \partial_{M}\right), \chi^{\pi}\right\rangle=d_{\pi} \int_{M} \sum_{j=0}^{m / 2} \frac{1}{j !} \hat{A}\left(R_{M}\right)_{m-2 j} \operatorname{Tr}\left(\pi\left(\left(\Omega^{(\sigma)}\right)^{j}\right)\right) .
$$

Proof. Let $E_{\pi}$ be the $\pi$-isotypic component of $\mathcal{E}$. Since the action of $\mathrm{Cl}_{\mathbb{C}}(M)$ on $\mathcal{E}$ commutes with the action of $\mathrm{SU}(N)$, the vector bundle $E_{\pi}$ is a Clifford submodule of $\mathcal{E}$. We show that the relative Chern character $\operatorname{ch}\left(E_{\pi} / \mathbb{S}\right)$ of the Clifford module $V_{\pi}$ is represented by $d_{\pi} \operatorname{Tr}\left(e^{\pi(\Omega)}\right)$. Then the assertion will follow from the index formula (2) for Clifford modules.

Let $p^{\pi}$ denote the projector $\int_{\mathrm{SU}(N)} \chi^{\pi}(g) g$ in the convolution algebra $L^{1}(\mathrm{SU}(N))$. Then $E_{\pi}$ is the image of $\pi^{P} \otimes \pi^{(\text {nat })}\left(p^{\pi}\right)$. When $\sigma$ is a section of $P$, the sections $\Gamma\left(\operatorname{dom} \sigma ; E_{\pi}\right)$ of $E_{\pi}$ over the domain of $\sigma$ can be identified with the space of functions

$$
x \mapsto\left(\sigma_{x}, f_{x}\right) \quad\left(f_{x} \in \rho \otimes \pi^{(\text {nat })}\left(p^{\pi}\right) \cdot L^{2}\left(\mathrm{PU}(N) ; V^{(\text {nat })}\right)\right)
$$

for $x \in \operatorname{dom} \sigma$. By the $\mathrm{SU}(N)$-invariance of $\nabla$ on $\mathcal{E}$, the associated curvature form of $E_{\pi}$ is given by $F \rho\left(p^{\pi}\right)$.

Applying the operator $S$, one sees that $\Gamma\left(\operatorname{dom} \sigma ; E_{\pi}\right)$ can also be identified with the space of functions $x \mapsto\left(\sigma_{x}, f_{x}\right)$ where $f_{x} \rho\left(p^{\pi}\right) \cdot L^{2}\left(\mathrm{SU}(N) ; V^{(\text {nat })}\right)^{\rho\left(\chi^{(\text {nat })}\right)}$. By the decomposition (14), the $\mathrm{SU}(N)$-space $\rho\left(p^{\pi}\right) \cdot L^{2}\left(\mathrm{SU}(N) ; V^{(\text {nat })}\right)^{\rho\left(\chi^{(\text {nat })}\right)}$ is isomorphic to the direct sum of $d_{\pi}$ copies of $V_{\pi} \otimes V^{(\text {nat })}$.

From (15), one sees that the action of the curvature form $F p^{\pi}$ on $E_{\pi}$ is given by $\pi\left(\Omega^{(\sigma)}\right) \otimes 1_{(\text {nat })}+1_{\pi} \otimes \pi^{(\text {nat })}\left(\Omega^{(\sigma)}\right)$. The term $1_{\pi} \otimes \pi^{(\text {nat })}\left(\Omega^{(\sigma)}\right)$ is the action of the spinor curvature. Hence the relative curvature form is given by $\pi\left(\Omega^{(\sigma)}\right) \otimes 1_{(\text {nat })}$, which proves the assertion.

Remark 5. Consider the case of $\pi=\pi^{\text {(nat) }}$ as in Example 4. Thus the left hand side of (18) is equal to the signature number of $M$. On the right hand side, the term $\operatorname{Tr}_{V^{(\text {nat })}}\left(\pi^{(\text {nat })}\left(\Omega^{j}\right)\right)$ is equal to the $j$-th component of the relative Chern character $\operatorname{ch}\left(\wedge^{*} T^{*} M / \mathbb{S}\right)$. Hence we recover the signature formula

$$
\sigma(M)=\int_{M} \hat{A}\left(R_{M}\right) \wedge \operatorname{ch}\left(\wedge^{*} \mathrm{~T}^{*} M / \mathbb{S}\right) .
$$

We obtain the following formula, whose conceptual meaning is given by (6) .

Corollary 6. The distribution $\operatorname{ind}_{\mathrm{SU}(N)}\left(\gamma, \not \partial_{M}\right)$ can be written as

$$
\left\langle\operatorname{ind}_{\mathrm{SU}(N)}\left(\gamma, \not \partial_{M}\right), \phi\right\rangle=\sum_{g \in Z(\mathrm{SU}(N))} \int_{M} \sum_{j=0}^{m / 2} \frac{1}{j !} \hat{A}\left(R_{M}\right)_{m-2 j}\left\langle T\left(\left(\Omega^{(\sigma)}\right)^{j}\right), \lambda_{g} \phi\right\rangle,
$$

where $\phi$ is any test function in $C^{\infty}(\mathrm{SU}(N))$.

Proof. Since both sides are invariant under conjugation for $\phi$, we may assume that $\phi$ is invariant under conjugation. By continuity and linearity, we may assume that $\phi$ is a character of some irreducible representation of $\mathrm{SU}(N)$. Then the assertion follows from Lemma 2 and Theorem 1.

Now, we can recover the fractional index formula (11) as a particular case of Corollary 6. 
Corollary 7 ([MMS06, Theorem 2; MMS08, Proposition 5 and Remark 1]). Let $\phi$ be a smooth function which agrees with the constant function 1 on a neighborhood of $e$ and satisfies $\operatorname{supp} \phi \cap Z(\mathrm{SU}(N))=\{e\}$. Then one has

$$
\left\langle\operatorname{ind}_{\mathrm{SU}(N)}\left(\gamma, \not_{M}\right), \phi\right\rangle=\int_{M} \hat{A}\left(R_{M}\right)
$$

Proof. For each $j$, the operator $T\left(\left(\Omega^{(\sigma)}\right)^{j}\right)$ is represented by a $\mathrm{SU}(N)$-biinvariant differential operator of degree $2 j$. Suppose that $\phi$ agrees with the constant function 1 on a neighborhood of $e$. Then one has

$$
\operatorname{Tr}(\rho(\phi))=\phi(e)=1, \quad \operatorname{Tr}\left(\rho(\phi) F^{j}\right)=\left\langle T\left(\left(\Omega^{(\sigma)}\right)^{j}\right), \phi\right\rangle=0 \quad(j>0) .
$$

Hence one has $\left\langle\operatorname{Tr}\left(\gamma e^{-F}\right), \phi\right\rangle=1$ in this case. Consequently one obtains

$$
\left\langle\operatorname{ind}_{\mathrm{SU}(N)}\left(\gamma, \not \partial_{M}\right), \phi\right\rangle=\int_{M} \hat{A}\left(R_{M}\right)
$$

which proves the assertion.

Remark 8. Since $\not \partial_{M}$ is formulated as a transversely elliptic operator on the $\mathrm{SU}(N)$ manifold $P$, the Kirillov type formulation of the equivariant index formula for such operators by Berline-Vergne BV96a BV96b might also be employed to prove the above result. Our presentation is based rather on the Atiyah-Segal-Singer type formulation of the equivariant index theorem.

\section{ACKNOWLEDGMENTS}

The author would like to thank R. Ponge for suggesting that he look at the papers of Mathai-Melrose-Singer and for many related discussions during the early stage of the research. He would also like to thank S. Neshveyev, Y. Oshima, R. Nest, and V. Mathai for fruitful conversations. He also benefited from exchanges with E. Meinrenken, S. Yamamoto, R. Tomatsu, N. Ozawa, M. Pichot, and M. Bischoff. Last but not least, he is deeply indebted to Y. Kawahigashi and D. E. Evans for their support throughout the period of the research.

\section{REFERENCES}

[AM] A. Alekseev and E. Meinrenken, Dirac structures and Dixmier-Douady bundles. Int. Math. Res. Not. 2012, no. 4, 904-956. MR2889163

[Ati74] M. F. Atiyah, Elliptic operators and compact groups, Lecture Notes in Mathematics, Vol. 401, Springer-Verlag, Berlin, 1974. MR0482866 (58:2910)

[BC00] P. Baum and A. Connes, Geometric K-theory for Lie groups and foliations, Enseign. Math. (2) 46 (2000), no. 1-2, 3-42. MR1769535 (2001i:19006)

[BG] M.-T. Benameur and A. Gorokhovsky, Local index theorem for projective families. Fields Inst. Commun., 61, Amer. Math. Soc., Providence, RI, 2011. MR2838679 (2012j:58023),

[BV96a] N. Berline and M. Vergne, The Chern character of a transversally elliptic symbol and the equivariant index, Invent. Math. 124 (1996), no. 1-3, 11-49. MR.1369410 (99a:58142)

[BV96b] N. Berline and M. Vergne, L'indice équivariant des opérateurs transversalement elliptiques, Invent. Math. 124 (1996), no. 1-3, 51-101. MR1369411 (99a:58143)

[Gof10] M. Goffeng, Projective pseudo-differential operators on infinite-dimensional Azumaya bundles (2010), available at http://arxiv.org/abs/1011.5800. 
[MMS05] V. Mathai, R. B. Melrose, and I. M. Singer, The index of projective families of elliptic operators, Geom. Topol. 9 (2005), 341-373 (electronic). MR2140985 (2005m:58044)

[MMS06] V. Mathai, R. B. Melrose, and I. M. Singer, Fractional analytic index, J. Differential Geom. 74 (2006), no. 2, 265-292. MR2258800(2007d:58034)

[MMS08] V. Mathai, R. B. Melrose, and I. M. Singer, Equivariant and fractional index of projective elliptic operators, J. Differential Geom. 78 (2008), no. 3, 465-473. MR 2396250 (2009a:58022)

[MMS09] V. Mathai, R. B. Melrose, and I. M. Singer, The index of projective families of elliptic operators: the decomposable case, Astérisque 328 (2009), 255-296. MR2674880

School of Mathematics, Cardiff University, Senghennydd Road, Cardiff, CF24 4AG, WALES, United Kingdom

Current address: Dipartimento di Matematica, Università degli Studi di Roma "Tor Vergata", Via della Ricerca Scientifica 1, 00133 Rome, Italy

E-mail address: makotoy@ms.u-tokyo.ac.jp 